\title{
Deux approches empiriques de la répartition spatiale des pluies: visualisation, simulation
}

\author{
Two empirical approaches to rainfall distribution \\ in space: display, simulation
}

\author{
T. Leviandier
}

CEMAGREF - Antony

Division Hydrologie-Hydraulique

\section{Introduction}

La représentation graphique est d'une utilité certaine dans l'étude de la répartition spatiale des pluies, tant du point de vue qualitatif (mise en évidence de bandes et de cellules, influence du relief) que du point de vue de l'estimation numérique. Ce dernier point devient moins vrai car, si dans les méthodes manuelles le tracé d'isohyètes peut servir à l'estimation ponctuelle ou au calcul de la moyenne spatiale, dans les méthodes automatiques, l'interpolation est préalable au dessin. Si la cartographie perd donc un peu comme justification l'estimation de valeurs ponctuelles, elle conserve l'intérêt d'une lecture facile et d'une représentation globale. Mais la lecture facile n'est même pas toujours nécessaire et on y renonce dans les représentations du relief en perspective (qu'on n'appellera peut-être plus cartographiques), le relief pouvant figurer toute autre grandeur scalaire que l'altitude. Sans chercher toutes les implications sousjacentes, on peut distinguer deux préoccupations différentes, la réalisation d'un document de synthèse, voire de prestige, et l'instrument de travail. C'est à ce deuxième type que nous nous intéressons désormais, cherchant une représentation globale permettant de détecter des structures avant de les modéliser, et ceci au moindre coût, en sacrifiant si besoin est la qualité de l'interpolation. La contrainte de moindre coût vient du souhait de faire un grand nombre de dessins pour appréhender l'évolution temporelle.

\section{Les lames d'eau sur des polygones de Thiessen}

Le dessin des lames d'eau sur des polygones de Thiessen est une réponse possible au problème posé. La méthode d'interpolation - égalité à la valeur observée au point le plus proche - est réputée à juste titre comme la plus mauvaise, encore que le calcul des valeurs moyennes soit en général très acceptable. En contrepartie, le cout du calcul et du dessin est très faible pour deux raisons : d'une part, il n'y a pas de véritable calcul d'interpolation, d'autre part, le dessin ne comporte pas de courbes. Or, en tracé automatique, les courbes sont des lignes brisées comportant un grand nombre de segments, ce qui entraîne un stockage important de résultats intermédiaires et un temps d'exécution assez long.

\section{Algorithme de calcul au tomatique des polygones de Thiessen}

L'algorithme reproduit la démarche manuelle de construction des médiatrices. Cependant, comme on s'interdit d'effacer physiquement, il faut mettre en mémoire tous les éléments du dessin et simuler l'effacement par modification des éléments déjà calculés. On peut facilement faire le dessin si on le désire, une fois tous les calculs faits.

Chaque polygone est décrit par ses arêtes, chaque arête par ses sommets et chaque sommet par ses coordonnées. Le polygone initial est identique au bassin tout entier, supposé convexe. Lorsqu'on introduit un nouveau poste, on recoupe certains des polygones précédents, créant ainsi de nouvelles arêtes et on constitue le nouveau polygone en rassemblant ces arêtes et éventuellement des arêtes du périmètre extérieur. Une économie de programmation consiste à ignorer les cas où cette partie du périmètre n'est pas connexe, mais il faut introduire les postes dans un ordre qui ne conduit pas à ce genre de situation, donc en commençant par les postes centraux (fig. 1).

Les problèmes de précision numérique compliquent notablement la programmation. En effet, on doit 


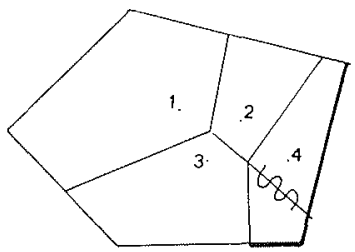

ou

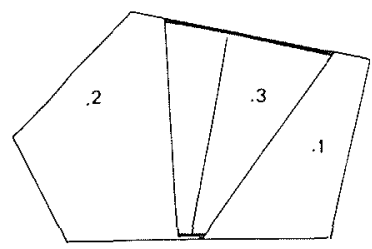

souvent tester si un segment est coupé par une droite et, comme par construction certains segments sont coupés en l'une de leurs extrêmités, il y a risque d'erreur.

\section{Algorithme de dessin en perspective des lames d'eau}

La perspective cavalière est utilisée mais pourrait être remplacée par toute autre perspective conservant les droites. Il s'agit de représenter des prismes qui peuvent se cacher mutuellement. Un prisme est représenté en perspective par un polygone et des arêtes verticales. On n'a pas à se préoccuper des arêtes horizontales de la base car seuls sont visibles les bords (non cachés) du bassin total et il suffit de représenter chaque face par les points de l'arête horizontale supérieure et la longueur de l'arête verticale, par exemple la première rencontrée dans l'ordre trigonométrique (fig. 2).

Il est à remarquer que si l'on ne change pas de réseau ni de perspective, mais seulement de hauteur d'eau, les relations d'antériorité des prismes ne changent pas et leur détermination peut donc être faite pour plusieurs événements sur le même réseau. La simulation des parties cachées consiste ensuite à chercher dans le plan du dessin la partie d'une figure intérieure à une ligne convexe.

Cette procédure s'applique aussi bien si l'image du prisme postérieur est déjà partiellement effacée. Quand au prisme antérieur, il semble préférable de considérer son image plane en rétablissant ses parties déjà masquées (sauf s'il est entièrement effacé).

Cette opération étant locale, puisque le cas des prismes mutuellement éloignés est rapidement traité, le temps de calcul ne croît pas rapidement avec le nombre de postes à traiter.

Sur IRIS 80 , le temps de calcul de 21 polygones est d'environ $7 \mathrm{~s}$, la détermination des relations d'antériorité des prismes est de $7 \mathrm{~s}$ et le calcul des parties visibles pour un épisode de $2 \mathrm{~s}$. Le temps de dessin effectif est bien sûr fonction de l'échelle et du matériel utilisé, en particulier pour une console de visualisation de la vitesse de transmission. Pour une exécution en temps partagé avec une vitesse de 300 bauds il est d'environ 45 s. Il serait de l'ordre de quelques secondes avec une console à 4800 bauds, avec lecture sur fichier des résultats préalablement calculés.

La rapidité de calcul pourrait être elle-même rapidement augmentée grâce à la propriété que les calculs d'effacement sur les paires de prismes peuvent être faits dans n'importe quel ordre, et donc en parallèle, sur des ordinateurs vectoriels.

A part son retentissement sur le coût, la rapidité du calcul n'est essentielle que pour le dessin animé en temps réel, ou dans une moindre mesure le film image par image à partir d'un écran. Elle a moins d'importance dans le cas d'un dessin sur papier ou directement sur film.

\section{Echelle de contraction des temps}

Le choix du pas de temps représenté, qui pose toujours un problème, devient crucial si on veut faire un film. Soit les deux exemples suivants: représenter une année, ou un épisode de 2 heures dans un film d'une minute à 18 images par seconde. Chaque image est alors représentative d'un intervalle de 8 heures environ dans le premier cas et de $4.5 \mathrm{~s}$ dans le second. $\mathrm{Si}$ on prend les moyennes sur ces intervalles, le premier film risque d'être assez chaotique, les images successives étant généralement très différentes, le second utilise des intervalles de temps inférieurs à la significativité des mesures sans commune mesure avec la synchronisation des différents postes. Or, rien n'interdit de prendre un intervalle de temps différent et en particulier plus grand que l'intervalle correspondant à l'image. On réalise ainsi une moyenne sur un intervalle glissant de durée fixe. Plusieurs essais peuvent être faits pour chercher la durée qui révèle le mieux une structure. Il est bien évident qu'au-delà d'une certaine valeur, on lisse trop les résultats.

\section{Applications}

La mise au point de cet outil étant récente, il n'a pas été assez exploité pour pouvoir donner autre chose que des exemples (fig. 3). L'un d'eux est relatif à des valeurs simulées (grâce à un modèle présenté en 1979 [1]) sur un réseau carroyé. La représentation graphique 
devrait permettre de compléter les tests numériques par des appréciations subjectives sur la ressemblance entre pluies observées et valeurs simulées. En particulier il serait intéressant de voir si la composition des aléas introduit dans le modèle peut engendrer des déplacements apparents globaux du champ de valeurs simulées, comparables au déplacement sur les valeurs réelles, ou s'il est nécessaire de les introduire explicitement. La partie numérique de la modélisation porte sur la reconstitution de corrélogrammes. Pour l'instant, on a seulement collationné des corrélogrammes obtenus avec différents paramètres du modèle sans établir de relation entre les uns et les autres, ni a fortiori fait de calage.
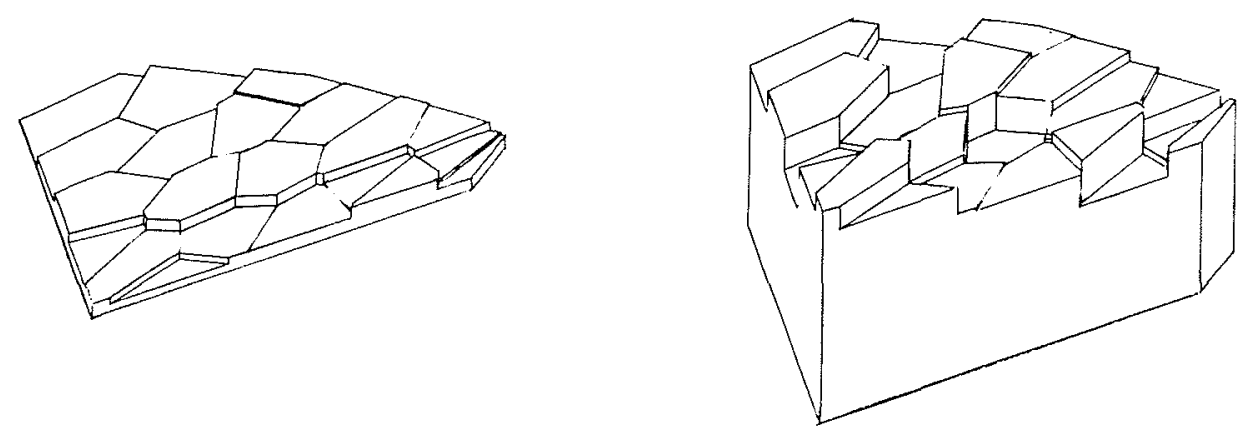

a) Hauteur de pluie sur $100 \mathrm{~km}^{2}$ pendant deux périodes de 6 heures consécutives.

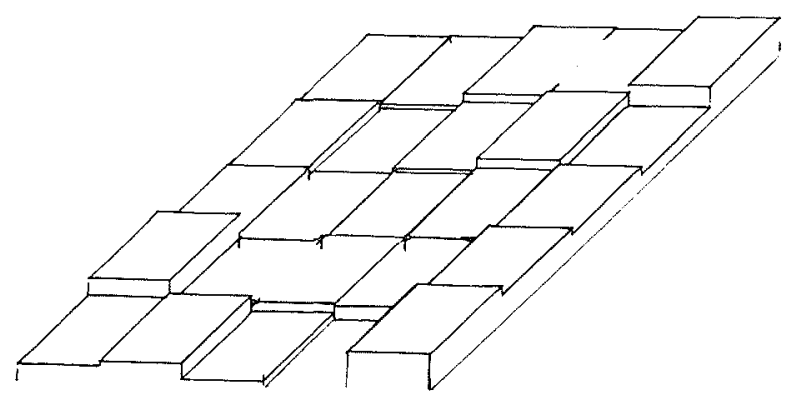

b) Valeurs obtenues par un modèle de simulation avec corrélation spatiale.

Figure 3 - Illustration de la méthode sur valeurs réelles et valeurs simulées.

\section{Bibliographie}

[1] T. LEVIANDIER. - Liaison spatiale entre postes pluviométriques, corrélation en fonction de la distance et du temps - Un modèle de simulation - La Météorologie, VI-20, mars et juin 1980. 